\title{
Corrigendum: Hypernetwork Construction and Feature Fusion Analysis Based on Sparse Group Lasso Method on fMRI Dataset
}

\author{
Yao Li ${ }^{1}$, Chao Sun ${ }^{1}$, Pengzu $\mathrm{Li}^{1}$, Yunpeng Zhao ${ }^{2}$, Godfred Kim Mensah ${ }^{1}$, Yong Xu ${ }^{3}$, \\ Hao Guo ${ }^{1 *}$ and Junjie Chen ${ }^{1 *}$ \\ ${ }^{1}$ College of Information and Computer, Taiyuan University of Technology, Taiyuan, China, ${ }^{2}$ College of Arts, Taiyuan University \\ of Technology, Taiyuan, China, ${ }^{3}$ Department of Psychiatry, First Hospital of Shanxi Medical University, Taiyuan, China
}

Keywords: hypernetwork, sparse group lasso, cluster coefficients based on pairs of nodes, multi-feature, classification, depression

\section{A Corrigendum on}

Hypernetwork Construction and Feature Fusion Analysis Based on Sparse Group Lasso Method on fMRI Dataset

by Li, Y., Sun, C., Li, P., Zhao, Y., Mensah, G. K., Xu, Y., et al. (2020). Front. Neurosci. 14:60. doi: 10.3389/fnins.2020.00060

\section{OPEN ACCESS}

Edited and reviewed by: Nathalie Just

INRA Centre Val de Loire, France

*Correspondence:

Hao Guo

feiyu_guo@sina.com

Junjie Chen

chenij@tyut.edu.cn

Specialty section:

This article was submitted to Brain Imaging Methods,

a section of the journal Frontiers in Neuroscience

Received: 13 February 2020 Accepted: 04 March 2020

Published: 02 April 2020

Citation:

Li Y, Sun C, Li P, Zhao Y, Mensah GK Xu Y, Guo H and Chen J (2020)

Corrigendum: Hypernetwork Construction and Feature Fusion Analysis Based on Sparse Group Lasso Method on fMRI Dataset.

Front. Neurosci. 14:243. doi: 10.3389/fnins.2020.00243
In the original article, there was an error in the title. Instead of "Hypernetwork Construction and Feature Fusion Analysis Based on Sparse Group Lasso Method on Functional fMRI Dataset" it should be "Hypernetwork Construction and Feature Fusion Analysis Based on Sparse Group Lasso Method on fMRI Dataset".

Additionally, in Equations (4) and (7) the parameters mentioned and its explanations were wrong. "n" should be superscript and not subscript. Also in Equation (4), "x" should not be in italics and " $\alpha$ " should be in italics. A correction has been made to the following sections:

The Materials and Methods section, subsection Construction of Hypernetwork, sub-subsection Sparse Linear Regression Model, paragraph 2:

"The average time series of $m$-th ROI for $n$-th subject, $\mathrm{x}_{m}^{n}=\mathrm{A}_{m}^{n} \alpha_{m}^{n}+\tau_{m}^{n}$, can be viewed as a response vector, which can be estimated as a linear combination of time series of other ROIs. The sparse linear regression model is specifically expressed as follows:

$$
\mathrm{x}_{m}^{n}=\mathrm{A}_{m}^{n} \alpha_{m}^{n}+\tau_{m}^{n}
$$

where $\mathrm{x}_{m}^{n}=\left[x_{m}^{n}(1) ; x_{m}^{n}(2) ; \ldots ; x_{m}^{n}(T)\right]$ refers to the average time series of the $m$-th ROI for $n$-th subjects, with $\mathrm{T}$ being the number of time points in the time series; $\mathrm{A}_{m}^{n}=$ $\left[\mathrm{x}_{1}^{n}, \ldots, \mathrm{x}_{m-1}^{n}, 0, \mathrm{x}_{m+1}^{n} \ldots, \mathrm{x}_{M}^{n}\right]$ denotes the data matrix of the $m$-th ROI (all the average time series except for the $m$-th brain region, and the average time series of the $m$-th ROI being set to 0 ); $\alpha_{m}^{n}=\left[\alpha_{1}^{n}, \ldots, \alpha_{m-1}^{n}, 0, \alpha_{m+1}^{n} \ldots, \alpha_{M}^{n}\right]$ denotes the coefficient vector that quantifies the degree of influence from the other ROI to the $m$-th ROI; and $\tau_{m}^{n}$ denotes a noise term, being Gaussian. The ROIs corresponding to the non-zero element in $\alpha_{m}^{n}$ are the ROIs interacting with the particular ROI; by contrast, the corresponding ROI of the zero element is conditionally independent with the $m$-th ROI."

The Materials and Methods section, subsection Construction of Hypernetwork, Construction of Hypernetworks Based on the Sparse Group Lasso Method, paragraph 3: 
"Similar to the gLasso method, clustering was adopted before creating the hyperedge, and then the sgLasso method was used to construct the hyperedge by solving the sparse linear regression model. The method is represented by the optimization objective function:

$$
\min _{\alpha_{m}}\left\|\mathrm{x}_{m}^{n}-\mathrm{A}_{m}^{n} \alpha_{m}^{n}\right\|_{2}+\lambda_{1}\left\|\alpha_{m}^{n}\right\|_{1}+\lambda_{2} \sum_{\mathrm{i}=1}^{\mathrm{k}}\left\|\alpha_{m}^{n} G_{i}\right\|_{2}
$$

$\alpha_{m}^{n} \quad$ is divided into $\mathrm{k}$ non-overlapping tree groups $\left(\alpha_{m}^{n} G_{1}, \alpha_{m}^{n} G_{2}, \ldots, \alpha_{m}^{n} G_{k}\right)$ by clustering, and $G_{i}$ is a node with tree structure. $\lambda_{1}$ and $\lambda_{2}$ are regression parameters, with $\lambda_{1}$ being used to adjust the sparsity of intra-groups to control the number of non-zero coefficients in non-zero groups, and $\lambda_{2}$ being used to adjust group-level sparsity (Yuan and Lin, 2006; Friedman et al., 2010) to control the number of groups with non-zero coefficients. This model is a combination of traditional lasso and gLasso. The gLasso estimate is obtained when $\lambda_{1}=0$, and

\section{REFERENCES}

Friedman, J., Hastie, T., and Tibshirani, R. (2010). Regularization paths for generalized linear models via coordinate descent. J. Statist. Softw. 33, 1-22. (accessed August 30, 2010).

Simon, N., Friedman, J., Hastie, T., and Robert, T. (2013). A sparse-group lasso. J. Computat. Graph. Statist. 22, 231-245.

Yuan, M., and Lin, Y. (2006). Model selection and estimation in regression with grouped variables. J. R. Statist. Soc. 68, 49-67. doi: 10.1111/j.1467-9868.2005.0 0532.x the lasso estimate is acquired when $\lambda_{2}=0$. It should be noted that the model looks somewhat similar to the elastic net model, but it is different because the $l_{2}$ penalty is not differentiated at 0 , so some groups are completely zeroed. However, in each non-zero group, it performs an elastic net fit (Simon et al., 2013). Like the gLasso method, a hypernetwork was constructed for each subject, where the ROI was regarded as the node, and the hyperedge comprised the $\mathrm{m}$-th ROI and the ROIs corresponding to the zero elements in $\alpha_{m}^{n}$. For each ROI, a set of hyperedges were produced by fixing the $\lambda_{2}$ value and varying the $\lambda_{1}$ value from 0.1 to 0.9 in increments of 0.1 . Finally, a hypernetwork is a $90^{*} 810$ matrix. In this experiment, the sgLasso method achieved the highest accuracy (87.12\%) of all three models, when $\lambda_{2}$ was equal to 0.4 . (see the Methodology Section about relative analysis)."

The authors apologize for these errors and state that this does not change the scientific conclusions of the article in any way. The original article has been updated.
Copyright (c) $2020 \mathrm{Li}$, Sun, Li, Zhao, Mensah, Xu, Guo and Chen. This is an openaccess article distributed under the terms of the Creative Commons Attribution License (CC BY). The use, distribution or reproduction in other forums is permitted, provided the original author(s) and the copyright owner(s) are credited and that the original publication in this journal is cited, in accordance with accepted academic practice. No use, distribution or reproduction is permitted which does not comply with these terms. 